\title{
Intelligent monitoring and health management technologies for metal-roof sheathing
}

\author{
YANG LiMan, FU ChenYao, SHI Yan ${ }^{*} \&$ SHEN DongKai \\ School of Automation Science and Electrical Engineering, Beihang University, Beijing 100191, China
}

Received June 20, 2018; accepted October 17, 2018; published online January 25, 2019

Citation: $\quad$ Yang L M, Fu C Y, Shi Y, et al. Intelligent monitoring and health management technologies for metal-roof sheathing. Sci China Tech Sci, 2019, 62:
1655-1656, https://doi.org/10.1007/s11431-018-9381-5

Metal-roof sheathings, generally made of cold-bending alloy-steel with insulation, sound-absorbing insulation and waterproof materials, are widely employed in large-span public buildings and industrial buildings such as sports stadiums, airport terminals, exhibition centers, large workshops and warehouses due to their excellent structural performance, unique appearance and installation convenience. However, metal roof sheathings work in complex natural environment all year round, suffering wind, rain, snow and thermal changes, which possibly leads to deformation, fatigue, crack, bolt looseness of fixed supports, etc. These problems inevitably weaken the wind-uplift resistance of metal roofs, resulting in severe damage even if the wind pressure does not exceed the design threshold $[1,2]$. In recent years, with the wide application of metal roofs, the accidents occur frequently. For example, in 2011 and 2013, the metal roof of Beijing Airport Terminal T3 was uplifted by the strong wind twice, causing huge economic losses and bad social impact. Thus, the health monitoring of metal roofs has attracted broad attention in the area of intelligent building and some new progress has been made in several critical technical problems such as wind pressure distribution and stress analysis of metal roofs, damage detection and data-driven health management, shown in Figure 1.

At present, the wind tunnel experiment and numerical simulation are common approaches for safety detection of metal roof in the early design stage of construction. By means of wind tunnel test for the scaling model, the de-

*Corresponding author (email: yesoyou@gmail.com) formation and stress distribution of metal roofs are estimated so that the weak areas can be reinforced in the process of construction. The numerical models are frequently carried out to simulate the complicated weather conditions like fluctuating wind, snow, rain and temperature variation. Thus, more precise analysis for deflection and stain characteristics of metal roofs and connected joints under various weather loads were studied in $[3,4]$, and the results can be used as a reference for pre-design and reliability analysis of buildings. Furthermore, modeling the wind pressure distribution is the most effective method of choosing the location of detection nodes for real-time monitoring system, because it is often impractical in large-span buildings to cover all a roof surface by laying sensors in quantity. According to the characteristics of wind load fluctuation and probability distribution, the pulsating airflow load spectrum of metal panel was constructed, and then wind pressure distribution law and turbulence characteristics of the roof air flow were studied to determine the dangerous areas that are likely to produce negative wind pressure or vortex. According to the simulation results, cornices, windward sides and the places of alien structure were dangerous areas, and based on which, the effective sensor placement rules can be proposed [5].

There have been many developments in safety detection approaches for metal roofs in service. The Detect Company in USA conducted leak point tests on roofing waterproof membranes through electronic leak detectors, and used infrared camera detection, atomic radiation and capacitance detection to perform further moisture analysis of metal roofs. Samsudin et al. [6] observed the impervious properties of 


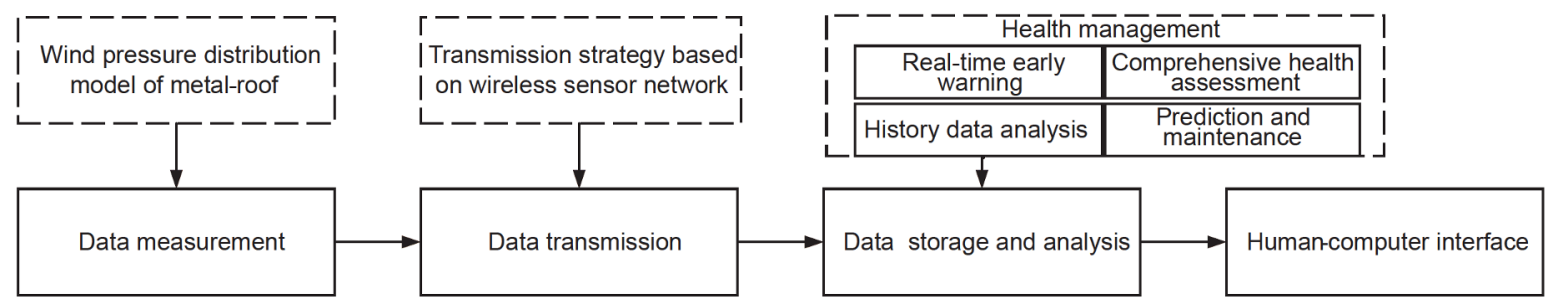

Figure 1 Key technology and system structure.

large-area-covered metal roofs in the city, and acquired the degraded state map of urban metal roofing materials by satellite multi-spectral remote detection. In recent years, some progress has been made on online-monitoring and data-driven health system based on wireless sensor network. The smart sensor nodes with embedded computing and wireless communication have the functions of sampling, processing, and transmission. Researches of wireless sensor network mainly focus on the issues of routing algorithms improvement, transmission strategies design, sensor information fusion, so as to reduce energy consumption as well as optimize the network stability [7]. For example, the working statuses of smart nodes are supposed to be controlled by the transmission strategy including sleep mode, normal mode, random inspection mode and on-line mode. Sometimes, it is inefficient to lay masses of sensors nodes to inspect largescaled structure. To tackle this problem, in the study of [8], a fast reconstruction model based on Levy-type for metal panels under wind pressure loads was established, through which the deflection distribution of the whole metal plate can be estimated by means of acquiring the deflection and location of limited points. In general, the common failure modes of metal-roof can be summarized as connection failure of panel and support, deformation of panel, lateral slip of panel, bolt looseness of support, etc., thus sensors such as strain gage, flexible sensor, ultrasonic sensor, laser sensor and camera can be adopted to inspect the status of metal roof. Based on measured data, weather information and design parameters, health management for metal-roof can be explored, which contains aspects of real-time status warning, long-term accumulated damage prediction and comprehensive evaluation. For real-time status warning, machine learning algorithms and big data analysis methods (ANN, SVM, association and clustering and etc.) can be used to process and fuse various data so as to identify damage classification and give fault early warning $[9,10]$. In my study of metal-roof monitoring, the method of decision tree is adopted to realize coupling of different measurements. In the early stage, some wind tunnel testing, destructive testing and numerical simulation are conducted to get the thresholds and training data. In service process, a mass of data can be acquired constantly and the training model can be iterated and updated. In addition, since the fatigue deformation is the primary failure mode, the life of the metal roof can be evaluated based on cumulative damage model and weather condition statistics. Because the structural health monitoring concerns the performance degradation along with time, time series analysis methods are proved successful for description of historical evolution trend. Furthermore, based on weather load spectrum statistics, long-term degradation trends and simulation model of metal-roof, the comprehensive performance of metal-roof can be evaluated.

To summarize, building intelligent monitoring and health management system is a promising approach to reveal the physical deterioration rules of metal-roof and prompt failure risk for condition-based maintenance. The monitoring system can not only avoid severe accidents and reduce economic losses, but also provide technical support for design optimization and construction by feedback of the analysis results of big data, thereby moving towards the target of smart buildings and smart cities.

1 Huang W, Dong W M. Introduction for metal roofing development and application classification. Archit Technol, 2015, 12: 1103-1107

2 Li Y, Wang Y, Chase J G, et al. Survey and introduction to the focused section on mechatronics for sustainable and resilient civil infrastructure. IEEE/ASME Trans Mechatron, 2013, 18: 1637-1646

3 Henderson D, Williams C, Gavanski E, et al. Failure mechanisms of roof sheathing under fluctuating wind loads. J Wind Eng Industrial Aerodyn, 2013, 114: 27-37

4 Song Y H, Yang L M, Wang Q S, et al. Deformation characteristic study of metal roof panel under wind uplift loading. Build Struct, 2015, 45: 87-91

5 An C, Yang L M, Li Y H, et al. A fast estimating model for deformation of metal roofing sheathing with wind pressure load. In: 2015 IEEE 10th Conference on 1ndustrial Electronics and Applications (1C1EA). Auckland: IEEE. 789-793

6 Samsudin S H, Shafri H Z M, Hamedianfar A. Development of spectral indices for roofing material condition status detection using field spectroscopy and WorldView-3 data. J Appl Remote Sens, 2016, 10: 025021

7 Sbarufatti C, Manes A, Giglio M. Application of sensor technologies for local and distributed structural health monitoring. Struct Control Health Monit, 2014, 21: 1057-1083

8 Yang L, Cui L, Li Y, et al. Inspection and reconstruction of metal-roof deformation under wind pressure based on bend sensors. Sensors, 2017, 17: 1054

9 Li X, Yu W, Villegas S. Structural health monitoring of building structures with online data mining methods. IEEE Syst J, 2016, 10: $1291-1300$

10 Kundu T, Khazaeli S, Ravandi A G, et al. The application of data mining and cloud computing techniques in data-driven models for structural health monitoring. In: Proceedings of SPIE, Health Monitoring of Structural and Biological Systems, Volume 9805. Las Vegas, 2016 